Article

\title{
Isometric Deformation of $(m, n)$-Type Helicoidal Surface in the Three Dimensional Euclidean Space
}

\author{
Erhan Güler (D) \\ Faculty of Sciences, Department of Mathematics, Bartın University, 74100 Bartın, Turkey; eguler@bartin.edu.tr; \\ Tel.: +90-378-5011000-1521
}

Received: 13 September 2018; Accepted: 26 October 2018; Published: 29 October 2018

\begin{abstract}
We consider a new kind of helicoidal surface for natural numbers $(m, n)$ in the three-dimensional Euclidean space. We study a helicoidal surface of value $(m, n)$, which is locally isometric to a rotational surface of value $(m, n)$. In addition, we calculate the Laplace-Beltrami operator of the rotational surface of value $(0,1)$.
\end{abstract}

Keywords: euclidean three-space; helicoidal surface of value $(m, n)$; rotational surface of value $(m, n)$; mean curvature; Gaussian curvature; Gauss map

\section{Introduction}

The notion of the finite-type immersion of submanifolds of a Euclidean space has been used in classifying and characterizing well-known Riemannian submanifolds [1]. Chen posed the problem of classifying the finite-type surfaces in the three-dimensional Euclidean space $\mathbb{E}^{3}$. Then, the theory of submanifolds of a finite type was studied by many geometers [1-21].

Lawson [22] gave the general definition of the Laplace-Beltrami operator in his lecture notes. Takahashi [23] stated that minimal surfaces and spheres are the only surfaces in $\mathbb{E}^{3}$ satisfying the condition $\Delta r=\lambda r, \lambda \in \mathbb{R}$. Ferrandez, Garay and Lucas [10] proved that the surfaces of $\mathbb{E}^{3}$ satisfying $\Delta H=A H, A \in \operatorname{Mat}(3,3)$ are either minimal, or an open piece of a sphere, or of a right circular cylinder. Choi and Kim [5] characterized the minimal helicoid in terms of a pointwise one-type Gauss map of the first kind.

Dillen, Pas and Verstraelen [7] proved that the only surfaces in $\mathbb{E}^{3}$ satisfying $\Delta r=A r+B$, $A \in \operatorname{Mat}(3,3), B \in \operatorname{Mat}(3,1)$ are the minimal surfaces, the spheres and the circular cylinders. Senoussi and Bekkar [24] studied helicoidal surfaces $M^{2}$ in $\mathbb{E}^{3}$, which are of the finite type in the sense of Chen with respect to the fundamental forms $I, I I$ and $I I I$.

The right helicoid (resp. catenoid) is the only ruled (resp. rotational) surface that is minimal in classical surface geometry in Euclidean space. If we focus on the ruled (helicoid) and rotational characters, we see Bour's theorem in [25]. The French mathematician Edmond Bour used the semi-geodesic coordinates and found a number of new cases of the deformation of surfaces in 1862 . He also gave in [25] a well-known theorem about the helicoidal and rotational surfaces.

Kenmotsu [26] focused on the surfaces of revolution with the prescribed mean curvature. Regarding helicoidal surfaces, do Carmo and Dajczer [3] proved that, by using a result of Bour [25], there exists a two-parameter family of helicoidal surfaces isometric to a given helicoidal surface. Hitt and Roussos [27] also studied the helicoidal surfaces with constant mean curvature. Ikawa $[14,15]$ determined pairs of surfaces by Bour's theorem. Güler [28] also studied the isometric helicoidal and rotational surfaces of value $m$. Güler and Yaylı [12] focused on the generalized Bour's theorem in three-space.

We consider a new kind of helicoidal surface of value $(m, n)$ in Euclidean three-space $\mathbb{E}^{3}$ in this paper. We give some basic notions of the three-dimensional Euclidean geometry in Section 2. In 
Section 3, we give the definition of a helicoidal surface of value $(m, n)$ and obtain isometric helicoidal and rotational surfaces of value $(m, n)$ (resp. of value $(0,1)$ in Section 4$)$ via Bour's theorem. We also calculate the mean curvature and the Gaussian curvature of the rotational surface of value $(0,1)$ in Section 4. Moreover, in Section 5, we calculate the Laplace-Beltrami operator of the rotational surface of value $(0,1)$. Finally, we give the rotational surface satisfying $\Delta \mathbf{R}_{0,1}=A \mathbf{R}_{0,1}$ in $\mathbb{E}^{3}$ in the last section.

\section{Preliminaries}

We shall identify a vector $(a, b, c)$ with its transpose. In this section, we will obtain the rotational and helicoidal surfaces in $\mathbb{E}^{3}$. The reader can find basic elements of differential geometry in $[29,30]$.

We define the rotational surface and helicoidal surface in $\mathbb{E}^{3}$. For an open interval $I \subset \mathbb{R}$, let $\gamma: I \longrightarrow \Pi$ be a curve in a plane $\Pi$, and let $\ell$ be a straight line in $\Pi$. A rotational surface in $\mathbb{E}^{3}$ is defined as a surface rotating the curve $\gamma$ around the line $\ell$ (these are called the profile curve and the axis, respectively). Suppose that when a profile curve $\gamma$ rotates around the axis $\ell$, it simultaneously displaces parallel lines orthogonal to the axis $\ell$, so that the speed of displacement is proportional to the speed of rotation. Then, the resulting surface is called the helicoidal surface with axis $\ell$ and pitch $a \in \mathbb{R}^{+}$.

Let $\ell$ be the line spanned by the vector $(0,0,1)$. The orthogonal matrix that fixes the above vector is given by

$$
M(\theta)=\left(\begin{array}{ccc}
\cos \theta & -\sin \theta & 0 \\
\sin \theta & \cos \theta & 0 \\
0 & 0 & 1
\end{array}\right), \theta \in \mathbb{R}
$$

The matrix $M$ is found by solving the following equations: $M \ell=\ell, M^{t} M=M M^{t}=I_{3}$, $\operatorname{det} M=1$, simultaneously. When the axis of rotation is $\ell$, there is a Euclidean transformation by which the axis is $\ell$ transformed to the $z$-axis of $\mathbb{E}^{3}$. The profile curve is given by $\gamma(r)=(r, 0, \varphi(r))$, where $\varphi(r): I \subset \mathbb{R} \longrightarrow \mathbb{R}$ is a differentiable function for all $r \in I$. A helicoidal surface in three-dimensional Euclidean space which is spanned by the vector $(0,0,1)$ with pitch $a$ is as follows

$$
\mathbf{H}(r, \theta)=M(\theta) \gamma(r)+a \theta \ell .
$$

When $a=0$, the helicoidal surface is just a rotational surface.

\section{Helicoidal Surfaces of Value $(m, n)$}

We define a new type of helicoidal surface. Using Bour's theorem on the helicoidal surface, we obtain an isometric rotational surface in this section.

Definition 1. A helicoidal surface of value $(m, n)$ is given by

$$
\mathbf{H}_{m, n}(r, \theta)=\mathbf{H}_{m}^{1}(r, \theta)+\mathbf{H}_{m, n}^{2}(r, \theta),
$$

where $m, n \in \mathbb{N}, \mathbf{H}_{m}^{1}(r, \theta)=\Re_{m}^{1} \cdot \gamma_{m}^{1}+\frac{1}{2} a \theta \ell, \mathbf{H}_{m, n}^{2}(r, \theta)=\Re_{m, n}^{2} \cdot \gamma_{m, n}^{2}+\frac{1}{2} a \theta \ell$, the rotating matrices $\Re_{m}^{1}$ and $\Re_{m, n}^{2}$ are

$$
\Re_{m}^{1}(\theta)=\left(\begin{array}{ccc}
\cos [(m+1) \theta] & \sin [(m+1) \theta] & 0 \\
-\sin [(m+1) \theta] & \cos [(m+1) \theta] & 0 \\
0 & 0 & 1
\end{array}\right)
$$

and

$$
\Re_{m, n}^{2}(\theta)=\left(\begin{array}{ccc}
\cos [(m+2 n+1) \theta] & -\sin [(m+2 n+1) \theta] & 0 \\
\sin [(m+2 n+1) \theta] & \cos [(m+2 n+1) \theta] & 0 \\
0 & 0 & 1
\end{array}\right)
$$


$\ell=(0,0,1)$ is the rotating axis, and the profile curves are

$$
\gamma_{m}^{1}(r)=\left(\frac{r^{m+1}}{m+1}, 0, \frac{1}{2} \varphi(r)\right), \gamma_{m, n}^{2}(r)=\left(-\frac{r^{m+2 n+1}}{m+2 n+1}, 0, \frac{1}{2} \varphi(r)\right),
$$

$m \in \mathbb{R}-\{-1\}$ in $\gamma_{m}^{1}, m \in \mathbb{R}-\{-1-2 n\}$ in $\gamma_{m, n}^{2}, r \in \mathbb{R}^{+}, 0 \leq \theta \leq 2 \pi$ and the pitch $a \in \mathbb{R}^{+}$. Since the helicoidal surface is given by rotating the profile curves $\gamma$ around the axis $\ell$ and simultaneously displacing parallel lines orthogonal to the axis $\ell$, the speed of displacement is proportional to the speed of rotation.

Next, we give a theorem about locally isometric helicoidal-rotational surfaces of value $(m, n)$.

Theorem 1. A helicoidal surface of value $(m, n)$

$$
\mathbf{H}_{m, n}(r, \theta)=\left(\begin{array}{c}
\frac{r^{m+1}}{m+1} \cos [(m+1) \theta]-\frac{r^{m+2 n+1}}{m+2 n+1} \cos [(m+2 n+1) \theta] \\
-\frac{r^{m+1}}{m+1} \sin [(m+1) \theta]-\frac{r^{m+2 n+1}}{m+2 n+1} \sin [(m+2 n+1) \theta] \\
\varphi(r)+a \theta
\end{array}\right)
$$

is isometric to the rotational surface of value $(m, n)$

$$
\mathbf{R}_{m, n}\left(r_{\mathbf{R}}, \theta_{\mathbf{R}}\right)=\left(\begin{array}{c}
\frac{r_{\mathbf{R}}^{m+1}}{m+1} \cos \left[(m+1) \theta_{\mathbf{R}}\right]-\frac{r_{\mathbf{R}}^{m+2 n+1}}{m+2 n+1} \cos \left[(m+2 n+1) \theta_{\mathbf{R}}\right] \\
-\frac{r_{\mathbf{R}}^{m+1}}{m+1} \sin \left[(m+1) \theta_{\mathbf{R}}\right]-\frac{r_{\mathbf{R}}^{m+2 n+1}}{m+2 n+1} \sin \left[(m+2 n+1) \theta_{\mathbf{R}}\right] \\
\varphi_{\mathbf{R}}\left(r_{\mathbf{R}}\right)
\end{array}\right)
$$

by Bour's theorem, where

$$
\begin{aligned}
& \varphi_{\mathbf{R}}^{\prime 2}= \frac{\left[\begin{array}{c}
G^{m+n-1}\left\{(m+3 n) G^{2 n}+2(m+2 n) G^{n} \cos [2(m+n+1) \theta]\right. \\
\left.-2 G^{n} \sin [2(m+n+1) \theta]+m+n\right\}
\end{array}\right]^{2} \operatorname{det} I}{\left[\begin{array}{c}
r^{2 m+2 n}\left\{(2 m+6 n) r^{4 n-1}+(4 m+8 n) r^{2 n} \cos [2(m+n+1) \theta]\right. \\
\left.+(2 m+2) r^{-1}\right\}
\end{array}\right]^{2} G} \\
&+\frac{4 r_{\mathbf{R}}^{4 m+4 n+2} \sin ^{2}\left[2(m+n+1) \theta_{\mathbf{R}}\right]}{r_{\mathbf{R}}^{2 m+2}\left(r_{\mathbf{R}}^{4 n}+2 r_{\mathbf{R}}^{2 n} \cos \left[2(m+n+1) \theta_{\mathbf{R}}\right]+1\right)} \\
&-r_{\mathbf{R}}^{2 m}\left[r_{\mathbf{R}}^{4 n}-2 r_{\mathbf{R}}^{2 n} \cos \left(2(m+n+1) \theta_{\mathbf{R}}\right)+1\right] \\
& r_{\mathbf{R}}=\sqrt{G}, \\
& \theta_{\mathbf{R}}=\theta+\int \frac{F}{G} d r, \\
& E=r^{2 m}\left(r^{4 n}-2 r^{2 n} \cos [2(m+n+1) \theta]+1\right)+\varphi^{\prime 2}, \\
& F=2 r^{2 m+2 n+1} \sin [2(m+n+1) \theta]+a \varphi^{\prime}, \\
& G=r^{2 m+2}\left(r^{4 n}+2 r^{2 n} \cos [2(m+n+1) \theta]+1\right)+a^{2}, \\
& \operatorname{det} I=E G-F^{2},
\end{aligned}
$$

$m \in \mathbb{R}-\{-1,-1-2 n\}, n \in \mathbb{R}, r \in \mathbb{R}^{+}, \theta \in I \subset \mathbb{R}$ and the pitch $a \in \mathbb{R}^{+}$.

Proof. The line element of the the helicoidal surface $\mathbf{H}_{m, n}(r, \theta)$ is

$$
\begin{aligned}
d s^{2}= & \left\{r^{2 m}\left(r^{4 n}-2 r^{2 n} \cos [2(m+n+1) \theta]+1\right)+\varphi^{\prime 2}\right\} d r^{2} \\
& +2\left\{2 r^{2 m+2 n+1} \sin [2(m+n+1) \theta]+a \varphi^{\prime}\right\} d r d \theta \\
& +\left\{r^{2 m+2}\left(r^{4 n}+2 r^{2 n} \cos [2(m+n+1) \theta]+1\right)+a^{2}\right\} d \theta^{2}
\end{aligned}
$$


Helices in $\mathbf{H}_{m, n}$ are curves defined by $r=$ const. Therefore curves in $\mathbf{H}_{m, n}$ that are orthogonal to helices supply the orthogonality condition $F d r+G d \theta=0$. Thus, we obtain $\theta=-\int \frac{F}{G} d r+c$, where $c$ is constant. Hence, if we put $\bar{\theta}=\theta+\int \frac{F}{G} d r$, then curves orthogonal to helices are given by $\bar{\theta}=$ const. Substituting the equation $d \theta=d \bar{\theta}-\frac{F}{G} d r$ into the line element (4), we have

$$
d s^{2}=\frac{Q}{G} d r^{2}+G d \bar{\theta}^{2}
$$

where $Q:=\operatorname{det} I$. Setting $\bar{r}:=\sqrt{\frac{Q}{G}} d r, \Omega(\bar{r}):=\sqrt{G},(5)$ becomes

$$
d s^{2}=d \bar{r}^{2}+\Omega^{2}(\bar{r}) d \bar{\theta}^{2} .
$$

The rotational surface (3) has the line element

$$
d s_{\mathbf{R}}^{2}=\frac{Q_{\mathbf{R}}}{G_{\mathbf{R}}} d r_{\mathbf{R}}^{2}+G_{\mathbf{R}} d \bar{\theta}_{\mathbf{R}}^{2}
$$

where

$$
\begin{aligned}
E_{\mathbf{R}} & =r_{\mathbf{R}}^{2 m}\left(r_{\mathbf{R}}^{4 n}+1-2 r_{\mathbf{R}}^{2 n} \cos \left[2(m+n+1) \theta_{\mathbf{R}}\right]\right)+\varphi_{\mathbf{R}}^{\prime 2} \\
F_{\mathbf{R}} & =2 r_{\mathbf{R}}^{2 m+2 n+1} \sin \left[2(m+n+1) \theta_{\mathbf{R}}\right] \\
G_{\mathbf{R}} & =r_{\mathbf{R}}^{2 m+2}\left(r_{\mathbf{R}}^{4 n}+1+2 r_{\mathbf{R}}^{2 n} \cos \left[2(m+n+1) \theta_{\mathbf{R}}\right]\right) .
\end{aligned}
$$

Again, setting $\bar{r}_{\mathbf{R}}:=\sqrt{\frac{Q_{\mathbf{R}}}{G_{\mathbf{R}}}} d r_{\mathbf{R}}, \Omega_{\mathbf{R}}\left(\bar{r}_{\mathbf{R}}\right):=\sqrt{G_{\mathbf{R}}}$, then (7) becomes

$$
d s_{\mathbf{R}}^{2}=d \bar{r}_{\mathbf{R}}^{2}+\Omega_{\mathbf{R}}^{2}\left(\bar{r}_{\mathbf{R}}\right) d \bar{\theta}_{\mathbf{R}}^{2}
$$

Comparing (6) with (8), if we take $\bar{r}=\bar{r}_{\mathbf{R}}, \bar{\theta}=\bar{\theta}_{\mathbf{R}}, \Omega(\bar{r})=\Omega_{\mathbf{R}}\left(\bar{r}_{\mathbf{R}}\right)$, then we have an isometry between $\mathbf{H}_{m, n}(r, \theta)$ and $\mathbf{R}_{m, n}\left(r_{\mathbf{R}}, \theta_{\mathbf{R}}\right)$. Therefore, it follows that

$$
\sqrt{\frac{Q}{G}} d r=\sqrt{\frac{Q_{\mathbf{R}}}{G_{\mathbf{R}}}} d r_{\mathbf{R}}
$$

Substituting the equation

$$
d r_{\mathbf{R}}=\sqrt{\frac{G_{\mathbf{R}}\left[(G)_{r}\right]^{2}}{\left[\left(G_{\mathbf{R}}\right)_{r}\right]^{2} G}} d r
$$

into (9), we get the function $\varphi_{\mathbf{R}}$.

\section{Helicoidal Surface of Value $(0,1)$}

We give the helicoidal surface of value $(0,1)$ using Bour's theorem in this section.

Proposition 1. A helicoidal surface of value $(0,1)$ : (see Figure 1)

$$
\mathbf{H}_{0,1}(r, \theta)=\left(\begin{array}{c}
r \cos (\theta)-\frac{r^{3}}{3} \cos (3 \theta) \\
-r \sin (\theta)-\frac{r^{3}}{3} \sin (3 \theta) \\
\varphi(r)+a \theta
\end{array}\right)
$$


is isometric to the rotational surface of value $(0,1)$

$$
\mathbf{R}_{0,1}\left(r_{\mathbf{R}}, \theta_{\mathbf{R}}\right)=\left(\begin{array}{c}
\sqrt{G} \cos \left[\left(\theta+\int \frac{F}{G} d r\right)\right]-\frac{1}{3} \sqrt{G^{3}} \cos \left[3\left(\theta+\int \frac{F}{G} d r\right)\right] \\
-\sqrt{G} \sin \left[\left(\theta+\int \frac{F}{G} d r\right)\right]-\frac{1}{3} \sqrt{G^{3}} \sin \left[3\left(\theta+\int \frac{F}{G} d r\right)\right] \\
\varphi_{\mathbf{R}}\left(r_{\mathbf{R}}\right)
\end{array}\right)
$$

where

$$
\begin{aligned}
\varphi_{\mathbf{R}}^{\prime 2}= & \frac{\left[\left\{3 G^{2}+4 G \cos (4 \theta)-2 G \sin (4 \theta)+1\right\}\right]^{2} \operatorname{det} I}{\left[r^{2}\left\{6 r^{3}+8 r^{2} \cos (4 \theta)+2 r^{-1}\right\}\right]^{2} G} \\
& +\frac{4 G^{3} \sin ^{2}\left[4\left(\theta+\int \frac{F}{G} d r\right)\right]}{G\left[G^{2}+2 G \cos \left[4\left(\theta+\int \frac{F}{G} d r\right)\right]+1\right]} \\
& -\left[G^{2}-2 G \cos \left[4\left(\theta+\int \frac{F}{G} d r\right)\right]+1\right] \\
E & =r^{4}-2 r^{2} \cos (4 \theta)+1+\varphi^{\prime 2} \\
F & =2 r^{3} \sin (4 \theta)+a \varphi^{\prime} \\
G & =r^{2}\left[r^{4}+2 r^{2} \cos (4 \theta)+1\right]+a^{2},
\end{aligned}
$$

$\operatorname{det} I=E G-F^{2}, r, a \in \mathbb{R}^{+}, 0 \leq \theta \leq 2 \pi$.

Proof. Taking $m=0, n=1$ in the previous theorem, we easily get the results.
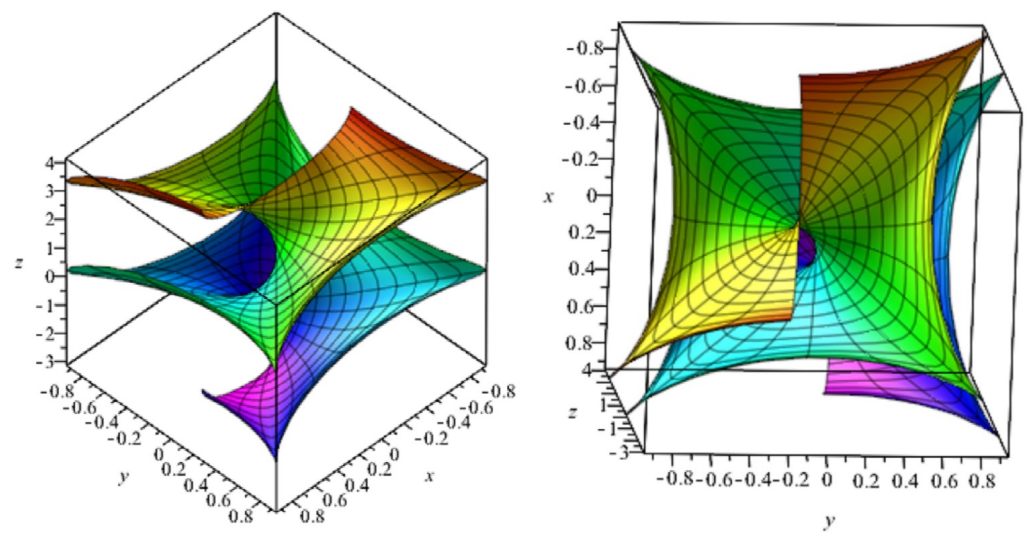

Figure 1. Two views of the helicoidal surface of value $(0,1), \varphi(r)=r^{2}$.

Corollary 1. When $a=0, \varphi(r)=r$ in $(10)$, we obtain a rotational surface of value $(0,1)$ (see Figure 2). 



Figure 2. Two views of the rotational surface of value $(0,1), \varphi(r)=r$.

Corollary 2. When $a=0$ and $\varphi(r)=r^{2} \cos (2 \theta)$ in (10), we have Enneper's minimal surface (see Figure 3).
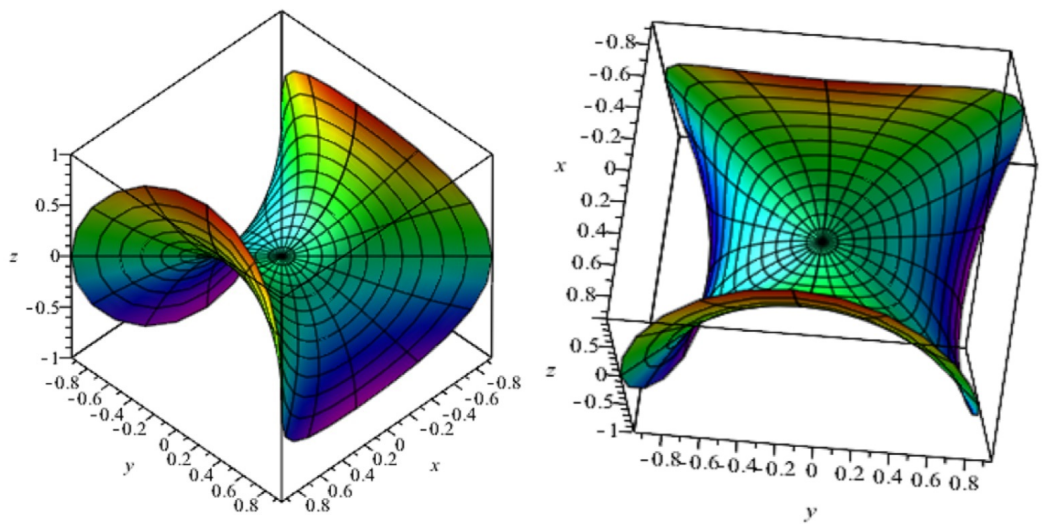

Figure 3. Two views of the Enneper minimal surface of value $(0,1), a=0, \varphi(r)=r^{2} \cos (2 \theta)$.

Proposition 2. The mean curvature and the Gaussian curvature of (10) are as follows

$$
\begin{aligned}
H= & \frac{1}{2(\operatorname{det} I)^{3 / 2}}\left\{r\left(r^{4}-1\right)\left(r^{6}+2 r^{4} \cos (4 \theta)+r^{2}+a^{2}\right) \varphi^{\prime \prime}\right. \\
& +r^{2}\left(3 r^{4}+2 r^{2} \cos (4 \theta)-1\right) \varphi^{3}-6 a r^{3} \sin (4 \theta) \varphi^{\prime 2} \\
& +\left[2 r^{2}\left(-5 r^{6}+r^{2}-3 a^{2}\right) \cos (4 \theta)+r^{10}-8 r^{6}+4 a^{2} r^{4}\right. \\
& \left.\left.-r^{2}-2 a^{2}\right] \varphi^{\prime}+2 a r\left(6 r^{6}-2 r^{2}+a^{2}\right) \sin (4 \theta)\right\}
\end{aligned}
$$

and

$$
\begin{aligned}
K= & \frac{1}{(\operatorname{det} I)^{2}}\left(\left\{\left[3 r^{11}+2 r^{5}\left(r^{4}-1\right) \cos (4 \theta)-4 r^{7}+r^{3}\right] \varphi^{\prime}\right.\right. \\
& \left.-2 a r^{5}\left(r^{4}-1\right) \sin (4 \theta)\right\} \varphi^{\prime \prime}+\left[-2 r^{6}\left(3 r^{4}+1\right)\right. \\
& \left.\left.-2 r^{4}\left(r^{6}-1\right) \cos (4 \theta)\right] \varphi^{\prime 2}-2 a r^{3}\left(3-11 r^{4}\right) \sin (4 \theta)\right] \varphi^{\prime} \\
& +4 a^{2} r^{2}\left(3 r^{4}-1\right) \cos (4 \theta)-a^{2}\left(9 r^{8}-2 r^{4}+1\right) .
\end{aligned}
$$

respectively, where $\operatorname{det} I=E G-F^{2}, \varphi^{\prime}=\frac{d \varphi}{d r}, r, a \in \mathbb{R}^{+}, 0 \leq \theta \leq 2 \pi$. 
Proof. Taking the differential with respect to $r, \theta$ to $\mathbf{H}_{0,1}$, we have

$$
\left(\mathbf{H}_{0,1}\right)_{r}=\left(\begin{array}{c}
\cos (\theta)-r^{2} \cos (3 \theta) \\
-\sin (\theta)-r^{2} \sin (3 \theta) \\
\varphi^{\prime}
\end{array}\right)
$$

and

$$
\left(\mathbf{H}_{0,1}\right)_{\theta}=\left(\begin{array}{c}
-r \sin (\theta)+r^{3} \sin (3 \theta) \\
-r \cos (\theta)-r^{3} \cos (3 \theta) \\
a
\end{array}\right)
$$

The coefficients of the first fundamental form of the surface are

$$
\begin{aligned}
E & =\left[r^{4}-2 r^{2} \cos (4 \theta)+1\right]+\varphi^{\prime 2} \\
F & =2 r^{3} \sin (4 \theta)+a \varphi^{\prime} \\
G & =r^{2}\left[r^{4}+2 r^{2} \cos (4 \theta)+1\right]+a^{2}
\end{aligned}
$$

Then, we get

$$
\begin{aligned}
\operatorname{det} I= & {\left[r^{6}+2 r^{4} \cos (4 \theta)+r^{2}\right] \varphi^{2}-4 a r^{3} \sin (4 \theta) \varphi^{\prime} } \\
& +r^{10}-2 r^{6}+a^{2} r^{4}-2 a^{2} r^{2} \cos (4 \theta)+r^{2}+a^{2}
\end{aligned}
$$

Using the second differentials

$$
\begin{gathered}
\left(\mathbf{H}_{0,1}\right)_{r r}=\left(\begin{array}{c}
-2 r \cos (3 \theta) \\
-2 r \sin (3 \theta) \\
\varphi^{\prime \prime}
\end{array}\right),\left(\mathbf{H}_{0,1}\right)_{r \theta}=\left(\begin{array}{c}
-\sin (\theta)+3 r^{2} \sin (3 \theta) \\
-\cos (\theta)-3 r^{2} \cos (3 \theta) \\
0
\end{array}\right) \\
\left(\mathbf{H}_{0,1}\right)_{\theta \theta}=\left(\begin{array}{c}
-r \cos (\theta)+3 r^{3} \cos (3 \theta) \\
r \sin (\theta)+3 r^{3} \sin (3 \theta) \\
0
\end{array}\right)
\end{gathered}
$$

and the Gauss map (the unit normal)

$$
e=\frac{1}{\sqrt{\operatorname{det} I}}\left(\begin{array}{c}
\left(r^{3} \cos (3 \theta)+r \cos (\theta)\right) \varphi^{\prime}-a\left(r^{2} \sin (3 \theta)+\sin (\theta)\right) \\
\left(r^{3} \sin (3 \theta)-r \sin (\theta)\right) \varphi^{\prime}+a\left(r^{2} \cos (3 \theta)-\cos (\theta)\right) \\
r^{5}-r
\end{array}\right)
$$

of the surface $\mathbf{H}_{0,1}$, we have the coefficients of the second fundamental form of the surface as follows

$$
\begin{aligned}
L & =\frac{1}{\sqrt{\operatorname{det} I}}\left[\left(r^{5}-r\right) \varphi^{\prime \prime}-r^{2}\left(r^{2}+\cos (4 \theta)\right) \varphi^{\prime}-2 a r \sin (4 \theta)\right] \\
M & =\frac{1}{\sqrt{\operatorname{det} I}}\left[2 r^{3} \sin (3 \theta) \varphi^{\prime}-3 a r^{4}+2 a r^{2} \cos (4 \theta)+a\right] \\
N & =\frac{1}{\sqrt{\operatorname{det} I}}\left[\left(3 r^{6}-r^{2}+2 r^{4} \cos (4 \theta)\right) \varphi^{\prime}-2 a r^{3} \sin (4 \theta)\right]
\end{aligned}
$$

Therefore, we can see the results easily. 
Corollary 3. If the helicoidal surface of value $(0,1)$ is minimal, then we get the differential equation as follows

$$
\begin{aligned}
& r\left(r^{4}-1\right)\left(r^{6}+2 r^{4} \cos (4 \theta)+r^{2}+a^{2}\right) \varphi^{\prime \prime} \\
& +r^{2}\left(3 r^{4}+2 r^{2} \cos (4 \theta)-1\right) \varphi^{3}-6 a r^{3} \sin (4 \theta) \varphi^{\prime 2} \\
& +\left[2 r^{2}\left(-5 r^{6}+r^{2}-3 a^{2}\right) \cos (4 \theta)+r^{10}-8 r^{6}+4 a^{2} r^{4}\right. \\
& \left.-r^{2}-2 a^{2}\right] \varphi^{\prime}+2 a r\left(6 r^{6}-2 r^{2}+a^{2}\right) \sin (4 \theta)=0 .
\end{aligned}
$$

The helicoidal surface of value $(1,1)$ is the same surface for value three in [28].

\section{Laplace-Beltrami Operator}

The Laplace-Beltrami operator of a smooth function $\phi=\phi(r, \theta) \mid \mathbf{D}\left(\mathbf{D} \subset \mathbb{R}^{3}\right)$ of class $C^{2}$ with respect to the first fundamental form of surface $\mathbf{M}$ is the operator $\Delta$, which is defined as follows

$$
\Delta \phi=\frac{1}{\sqrt{g}} \sum_{i, j=1}^{2} \frac{\partial}{\partial x^{i}}\left(\sqrt{g} g^{i j} \frac{\partial \phi}{\partial x^{j}}\right)
$$

where $\left(g^{i j}\right)=\left(g_{k l}\right)^{-1}$ and $g=\operatorname{det}\left(g_{i j}\right)$. Clearly, we write $\Delta \phi$ as follows

$$
\Delta \phi=\frac{1}{\sqrt{g}}\left[\begin{array}{c}
\frac{\partial}{\partial x^{1}}\left(\sqrt{g} g^{11} \frac{\partial \phi}{\partial x^{1}}\right)-\frac{\partial}{\partial x^{1}}\left(\sqrt{g} g^{12} \frac{\partial \phi}{\partial x^{2}}\right) \\
-\frac{\partial}{\partial x^{2}}\left(\sqrt{g} g^{21} \frac{\partial \phi}{\partial x^{1}}\right)+\frac{\partial}{\partial x^{2}}\left(\sqrt{g} g^{22} \frac{\partial \phi}{\partial x^{2}}\right)
\end{array}\right] .
$$

Using a more transparent notation, we get

$$
\Delta \phi=\frac{1}{\sqrt{\operatorname{det} I}}\left[\frac{\partial}{\partial u}\left(\frac{G \phi_{u}-F \phi_{v}}{\sqrt{\operatorname{det} I}}\right)-\frac{\partial}{\partial v}\left(\frac{F \phi_{u}-E \phi_{v}}{\sqrt{\operatorname{det} I}}\right)\right]
$$

where $g=\operatorname{det}\left(g_{i j}\right)=\operatorname{det} I$.

Now, we consider the rotational surface

$$
\mathbf{R}_{0,1}(r, \theta)=\left(\begin{array}{c}
r \cos (\theta)-\frac{r^{3}}{3} \cos (3 \theta) \\
-r \sin (\theta)-\frac{r^{3}}{3} \sin (3 \theta) \\
\varphi(r)
\end{array}\right)
$$

The first fundamental matrix of the surface is as follows

$$
I=\left(\begin{array}{cc}
r^{4}-2 r^{2} \cos (4 \theta)+1+\varphi^{\prime 2} & 2 r^{3} \sin (4 \theta) \\
2 r^{3} \sin (4 \theta) & r^{2}\left(r^{4}+2 r^{2} \cos (4 \theta)+1\right)
\end{array}\right) .
$$

The inverse matrix of $I$ is as follows

$$
\left(I^{-1}\right)=\frac{1}{\operatorname{det} I}\left(\begin{array}{cc}
r^{2}\left(r^{4}+2 r^{2} \cos 4 \theta+1\right) & -2 r^{3} \sin 4 \theta \\
-2 r^{3} \sin 4 \theta & r^{4}-2 r^{2} \cos 4 \theta+1+\varphi^{\prime 2}
\end{array}\right)
$$

where

$$
\operatorname{det} I=r^{2}\left(\left(r^{4}-2\right) r^{4}+\left(r^{4}+2 r^{2} \cos 4 \theta+1\right) \varphi^{2}+1\right) .
$$

The Laplace-Beltrami operator $\Delta \mathbf{R}_{0,1}$ of a rotational surface $\mathbf{R}_{0,1}$ is given by

$$
\Delta \mathbf{R}=\frac{1}{\sqrt{\operatorname{det} I}}\left(\frac{\partial}{\partial r} \mathbf{U}-\frac{\partial}{\partial \theta} \mathbf{V}\right)
$$


where

$$
\mathbf{U}=\frac{G \mathbf{R}_{r}-F \mathbf{R}_{\theta}}{\sqrt{\operatorname{det} I}}, \mathbf{V}=\frac{F \mathbf{R}_{r}-E \mathbf{R}_{\theta}}{\sqrt{\operatorname{det} I}} .
$$

Then, we obtain the following results

$$
\begin{aligned}
& \mathbf{U}=\frac{1}{\sqrt{\operatorname{det} I}}\left(\begin{array}{c}
-r^{2}\left(r^{4}-1\right)\left(\cos \theta+r^{2} \cos 3 \theta\right) \\
r^{2}\left(r^{4}-1\right)\left(\sin \theta-r^{2} \sin 3 \theta\right) \\
r^{2} \varphi^{\prime}\left(r^{4}+2 r^{2} \cos (4 \theta)+1\right)
\end{array}\right), \\
& \mathbf{V}=\frac{1}{\sqrt{\operatorname{det} I}}\left(\begin{array}{c}
r\left(\left(1+\varphi^{\prime 2}-r^{4}\right) \sin \theta+r^{2}\left(1-r^{4}-\varphi^{2 \prime}\right) \sin 3 \theta\right) \\
r\left(\left(1+\varphi^{2 \prime}-r^{4}\right) \cos \theta-r^{2}\left(1-r^{4}-\varphi^{\prime 2}\right) \cos 3 \theta\right) \\
2 r^{3} \varphi^{\prime} \sin (4 \theta)
\end{array}\right) .
\end{aligned}
$$

Using differentials of $r, \theta$ on $\mathbf{U}, \mathbf{V}$, respectively, we get

$$
\begin{aligned}
\Delta^{I} \mathbf{R} & =\frac{1}{\sqrt{\operatorname{det} I}}\left(\frac{\partial}{\partial r}(\mathbf{U})-\frac{\partial}{\partial \theta}(\mathbf{V})\right) \\
& =\frac{r^{3}}{(\operatorname{det} I)^{2}}\left(\begin{array}{c}
J_{1} \varphi^{\prime} \varphi^{\prime \prime}+J_{2} \varphi^{\prime 4}+J_{3} \varphi^{\prime 2}+J_{4} \\
T_{1} \varphi^{\prime} \varphi^{\prime \prime}+T_{2} \varphi^{\prime 4}+T_{3} \varphi^{\prime 2}+T_{4} \\
Q_{1} \varphi^{\prime 3} \varphi^{\prime \prime}+Q_{2} \varphi^{\prime 2} \varphi^{\prime \prime}+Q_{3} \varphi^{\prime} \varphi^{\prime \prime}+Q_{4} \varphi^{\prime 3}+Q_{5}
\end{array}\right),
\end{aligned}
$$

where

$$
\begin{aligned}
& J_{1}=r\left(1+r^{4}-2 r^{8}\right) \cos \theta+r^{3}\left(2-r^{4}-r^{8}\right) \cos 3 \theta+r^{3}\left(1-r^{4}\right) \cos 5 \theta+r^{5}\left(1-r^{4}\right) \cos 7 \theta, \\
& J_{2}=-\cos \theta+4 r^{4} \cos \theta+3 r^{6} \cos 3 \theta+r^{2} \cos 5 \theta+r^{4} \cos 7 \theta \\
& J_{3}=\quad\left(-3+r^{4}+14 r^{8}\right) \cos \theta+r^{2}\left(-6+7 r^{4}+11 r^{8}\right) \cos 3 \theta+r^{2}\left(1+3 r^{4}\right) \cos 5 \theta+ \\
& r^{4}\left(-3+7 r^{4}\right) \cos 7 \theta \\
& J_{4}=2\left(-1+3 r^{4}-3 r^{8}+r^{12}\right) \cos \theta+6 r^{2}\left(-1+3 r^{4}-3 r^{8}+r^{12}\right) \cos 3 \theta \\
& T_{1}=r\left(1-r^{8}\right) \sin \theta+r^{3}\left(-1+r^{8}\right) \sin 3 \theta+2 r^{3}\left(1-r^{4}\right) \cos 4 \theta \sin \theta+2 r^{5}\left(-1+r^{4}\right) \sin 3 \theta \cos 4 \theta, \\
& T_{2}=\left(-1+4 r^{4}\right) \cos \theta+3 r^{6} \cos 3 \theta+r^{2} \cos 5 \theta+r^{4} \cos 7 \theta \\
& T_{3}=\quad\left(-2-3 r^{4}+5 r^{8}\right) \cos \theta+\left(-1+6 r^{4}+3 r^{8}\right) \sin \theta+r^{2}\left(3-6 r^{4}-5 r^{8}\right) \sin 3 \theta+ \\
& 3 r^{2}\left(-1-r^{4}+2 r^{8}\right) \cos 3 \theta+r^{2}\left(1-r^{4}\right) \cos 5 \theta+8 r^{6} \sin \theta \cos 4 \theta+4 r^{4}\left(1-3 r^{4}\right) \sin 3 \theta \cos 4 \theta \\
& +r^{4}\left(-1+r^{4}\right) \cos 7 \theta, \\
& T_{4}=\left(-1+3 r^{4}-3 r^{8}+r^{12}\right)(\cos \theta+\sin \theta+\cos 3 \theta-\sin 3 \theta), \\
& Q_{1}=r+4 r^{3}+2 r^{5}+r^{9}+4 r^{5}\left(r^{2}+\cos ^{2} 4 \theta\right) \cos 4 \theta \\
& Q_{2}=-r-2 r^{5}-r^{9}-4 r^{3}\left(1+r^{4}+r^{2} \cos 4 \theta\right) \cos 4 \theta \\
& Q_{3}=r-r^{5}-r^{9}+r^{13}+2 r^{3}\left(1-2 r^{4}+r^{8}\right) \cos 4 \theta \\
& Q_{4}=1-4 r^{4}+3 r^{8}-2 r^{2}\left(1-r^{4}\right) \cos 4 \theta \\
& Q_{5}=1+7 r^{4}-9 r^{8}+r^{12}+r^{2}\left(-2+12 r^{4}-10 r^{8}\right) \cos 4 \theta .
\end{aligned}
$$

Remark 1. When the rotational surface $\mathbf{R}_{0,1}$ has the equation $\Delta \mathbf{R}_{0,1}=\mathbf{0}$, we have to solve the system of equations as follows

$$
\begin{aligned}
J_{1} \varphi^{\prime} \varphi^{\prime \prime}+J_{2} \varphi^{\prime 4}+J_{3} \varphi^{2}+J_{4} & =0, \\
T_{1} \varphi^{\prime} \varphi^{\prime \prime}+T_{2} \varphi^{4}+T_{3} \varphi^{\prime 2}+T_{4} & =0, \\
Q_{1} \varphi^{\prime 3} \varphi^{\prime \prime}+Q_{2} \varphi^{\prime 2} \varphi^{\prime \prime}+Q_{3} \varphi^{\prime} \varphi^{\prime \prime}+Q_{4} \varphi^{\prime 3}+Q_{5} & =0 .
\end{aligned}
$$

here, finding the function $\varphi$ is a problem.

Corollary 4. When $\varphi=c=$ const., then we get

$$
\Delta \mathbf{R}_{\mathbf{0 , 1}}=\frac{-1+3 r^{4}-3 r^{8}+r^{12}}{r^{2}\left[\left(r^{4}-2\right) r^{4}+1\right]}\left(\begin{array}{c}
2 \cos \theta+6 r^{2} \cos 3 \theta \\
\cos \theta+\sin \theta+3 r^{2}(\cos 3 \theta-\sin 3 \theta) \\
0
\end{array}\right) .
$$


6. Rotational Surface Satisfying $\Delta \mathbf{R}_{0,1}=A \mathbf{R}_{0,1}$ in $\mathbb{E}^{3}$

Theorem 2. Let $\mathbf{R}_{0,1}: M^{2} \longrightarrow \mathbb{E}^{3}$ be an isometric immersion given by (15). Then, $\Delta \mathbf{R}_{0,1}=A \mathbf{R}_{0,1}$ if and only if $M^{2}$ has zero mean curvature.

Proof. The Gauss map of the rotational surface $\mathbf{R}_{0,1}$ is

$$
e=\frac{1}{W}\left(\begin{array}{c}
\left(\cos \theta+r^{2} \cos 3 \theta\right) \varphi^{\prime} \\
\left(-\sin \theta+r^{2} \sin 3 \theta\right) \varphi^{\prime} \\
-1+r^{4}
\end{array}\right)
$$

where

$$
W=\sqrt{\left(r^{4}-2\right) r^{4}+\left(r^{4}+2 r^{2} \cos 4 \theta+1\right) \varphi^{\prime 2}+1} .
$$

We use

$$
-2 \mathrm{He}=A \mathbf{R}_{\mathbf{0 , 1}},
$$

where $A=\left(a_{i j}\right)$ is a $3 \times 3$ matrix. The equation $\Delta \mathbf{R}_{\mathbf{0}, \mathbf{1}}=A \mathbf{R}_{\mathbf{0}, \mathbf{1}}$ by means of

$$
I=\left(\begin{array}{cc}
r^{4}-2 r^{2} \cos (4 \theta)+1+\varphi^{\prime 2} & 2 r^{3} \sin (4 \theta) \\
2 r^{3} \sin (4 \theta) & r^{2}\left(r^{4}+2 r^{2} \cos (4 \theta)+1\right)
\end{array}\right) .
$$

and $\Delta \mathbf{R}_{\mathbf{0}, \mathbf{1}}=-2 \mathrm{He}$ give rise to the following system of ODEs

$$
\begin{gathered}
\Omega\left(\cos \theta+r^{2} \cos 3 \theta\right) \varphi^{\prime}-a_{11}\left(r \cos (\theta)-\frac{r^{3}}{3} \cos (3 \theta)\right)-a_{12}\left(-r \sin (\theta)-\frac{r^{3}}{3} \sin (3 \theta)\right)=\varphi a_{13} \\
\Omega\left(-\sin \theta+r^{2} \sin 3 \theta\right) \varphi^{\prime}-a_{21}\left(r \cos (\theta)-\frac{r^{3}}{3} \cos (3 \theta)\right)-a_{22}\left(-r \sin (\theta)-\frac{r^{3}}{3} \sin (3 \theta)\right)=\varphi a_{23} \\
\Omega\left(-1+r^{4}\right)=a_{31}\left(r \cos (\theta)-\frac{r^{3}}{3} \cos (3 \theta)\right)+a_{32}\left(-r \sin (\theta)-\frac{r^{3}}{3} \sin (3 \theta)\right)+\varphi a_{33}
\end{gathered}
$$

where $\Omega(r)=\frac{2 H}{W}$. Differentiating the ODEs with respect to $\theta$, we have

$$
a_{13}=a_{23}=a_{33}=0, \Omega(r)=0 .
$$

From (17), we get

$$
\begin{aligned}
-a_{11}\left(r \cos (\theta)-\frac{r^{3}}{3} \cos (3 \theta)\right)-a_{12}\left(-r \sin (\theta)-\frac{r^{3}}{3} \sin (3 \theta)\right) & =0, \\
-a_{21}\left(r \cos (\theta)-\frac{r^{3}}{3} \cos (3 \theta)\right)-a_{22}\left(-r \sin (\theta)-\frac{r^{3}}{3} \sin (3 \theta)\right) & =0, \\
a_{31}\left(r \cos (\theta)-\frac{r^{3}}{3} \cos (3 \theta)\right)+a_{32}\left(-r \sin (\theta)-\frac{r^{3}}{3} \sin (3 \theta)\right) & =0 .
\end{aligned}
$$

here, cos and $\sin$ are linearly independent functions of $\theta$, then we have that $a_{i j}=0$. From $\Omega(r)=\frac{2 H}{W}$, we obtain $H=0$. Finally, $\mathbf{R}_{\mathbf{0}, \mathbf{1}}$ is a minimal rotational hypersurface.

Author Contributions: The author gave the idea for the isometric deformation problem of an $(m, n)$-type helicoidal surface in three-space. Then, he checked and polished the draft.

Funding: This research received no external funding.

Conflicts of Interest: The author declares that there is no conflict of interest regarding the publication of this paper. 


\section{References}

1. Chen, B.Y. Total Mean Curvature and Submanifolds of Finite Type; World Scientific: Singapore, 1984.

2. Arslan, K.; Kılıç Bayram, B.; Bulca, B.; Öztürk, G. Generalized Rotation Surfaces in $\mathbb{E}^{4}$. Results Math. 2012, 61, 315-327. [CrossRef]

3. Do Carmo, M.; Dajczer, M. Helicoidal surfaces with constant mean curvature. Tohoku Math. J. 1982, 34, 351-367. [CrossRef]

4. Chen, B.Y.; Choi, M.; Kim, Y.H. Surfaces of revolution with pointwise 1-type Gauss map. Korean Math. Soc. 2005, 42, 447-455. [CrossRef]

5. Choi, M.; Kim, Y.H. Characterization of the helicoid as ruled surfaces with pointwise 1-type Gauss map. Bull. Korean Math. Soc. 2001, 38, 753-761.

6. Choi, M.; Yoon, D.W. Helicoidal surfaces of the third fundamental form in Minkowski 3-space. Bull. Korean Math. Soc. 2015, 52, 1569-1578. [CrossRef]

7. Dillen, F.; Pas, J.; Verstraelen, L. On surfaces of finite type in Euclidean 3-space. Kodai Math. J. 1990, 13, $10-21$. [CrossRef]

8. Dursun, U.; Turgay, N.C. Minimal and pseudo-umbilical rotational surfaces in Euclidean space $\mathbb{E}^{4}$. Mediterr. J. Math. 2013, 10, 497-506. [CrossRef]

9. Kim, Y.H.; Turgay, N.C. Surfaces in $\mathbb{E}^{3}$ with $L_{1}$-pointwise 1-type Gauss map. Bull. Korean Math. Soc. 2013, 50, 935-949. [CrossRef]

10. Ferrandez, A.; Garay, O.J.; Lucas, P. On a Certain Class of Conformally at Euclidean Hypersurfaces. In Global Analysis and Global Differential Geometry; Springer: Berlin, Germany, 1990; pp. 48-54.

11. Güler, E.; Turgut Vanl, A. Bour's theorem in Minkowski 3-space. J. Math. Kyoto Univ. 2006, 46, 47-63. [CrossRef]

12. Güler, E.; Yaylı, Y. Generalized Bour theorem. Kuwait J. Sci. 2015, 42, 79-90.

13. Güler, E.; Yaylı, Y.; Hacı salihoğlu, H.H. Bour's theorem on the Gauss map in 3-Euclidean space. Hacettepe J. Math. Stat. 2010, 39, 515-525.

14. Ikawa, T. Bour's theorem and Gauss map. Yokohama Math. J. 2000, 48, 173-180.

15. Ikawa, T. Bour's theorem in Minkowski geometry. Tokyo J. Math. 2001, 24, 377-394. [CrossRef]

16. Ji, F.; Kim, Y.H. Mean curvatures and Gauss maps of a pair of isometric helicoidal and rotation surfaces in Minkowski 3-space. J. Math. Anal. Appl. 2010, 368, 623-635. [CrossRef]

17. Ji, F.; Kim, Y.H. Isometries between minimal helicoidal surfaces and rotation surfaces in Minkowski space. Appl. Math. Comput. 2013, 220,1-11. [CrossRef]

18. The Hieu, D.; Ngoc Thang, N. Bour's theorem in 4-dimensional Euclidean space. Bull. Korean Math. Soc. 2017, 54, 2081-2089.

19. Ganchev, G.; Milousheva, V. General rotational surfaces in the 4-dimensional Minkowski space. Turk. J. Math. 2014, 38, 883-895. [CrossRef]

20. Güler, E.; Magid, M.; Yaylı, Y. Laplace Beltrami operator of a helicoidal hypersurface in four space. J. Geom. Sym. Phys. 2016, 41, 77-95. [CrossRef]

21. Güler, E.; Hacısalihoğlu, H.H.; Kim, Y.H. The Gauss Map and the third Laplace-Beltrami operator of the rotational hypersurface in 4-Space. Symmetry 2018, 10, 398. [CrossRef]

22. Lawson, H.B. Lectures on Minimal Submanifolds, 2nd ed.; Mathematics Lecture Series, 9; Publish or Perish, Inc.: Wilmington, NC, USA, 1980; Volume I.

23. Takahashi, T. Minimal immersions of Riemannian manifolds. J. Math. Soc. Jpn. 1966, 18, 380-385. [CrossRef]

24. Senoussi, B.; Bekkar, M. Helicoidal surfaces with $\Delta^{J} r=A r$ in 3-dimensional Euclidean space. Stud. Univ. Babeş-Bolyai Math. 2015, 60, 437-448.

25. Bour, E. Théorie de la déformation des surfaces. J. Êcole Imperiale Polytech. 1862, 22-39, 1-148.

26. Kenmotsu, K. Surfaces of revolution with prescribed mean curvature. Tohôku Math. J. 1980, 32, 147-153. [CrossRef]

27. Hitt, L.; Roussos, I. Computer graphics of helicoidal surfaces with constant mean curvature. An. Acad. Brasil. Ciênc. 1991, 63, 211-228.

28. Güler, E. A new kind helicoidal surface of value m. Int. Elec. J. Geom. 2014, 7, 154-162. 
29. Eisenhart, L. A Treatise on the Differential Geometry of Curves and Surfaces; Palermo 41 Ginn and Company: Orland, MA, USA, 1909.

30. Spivac, M. A Comprehensive Introduction to Differential Geometry III; Interscience: New York, NY, USA, 1969.

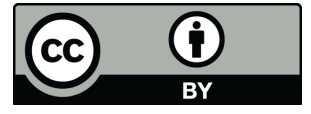

(C) 2018 by the authors. Licensee MDPI, Basel, Switzerland. This article is an open access article distributed under the terms and conditions of the Creative Commons Attribution (CC BY) license (http://creativecommons.org/licenses/by/4.0/). 